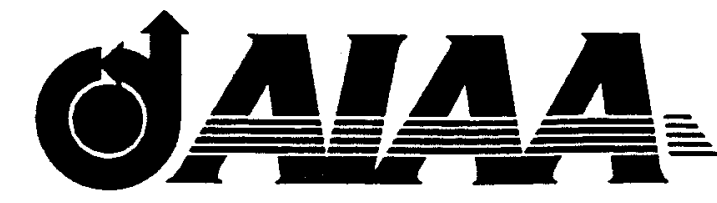

AIAA 2005-0000

\title{
Simulation of 3-D Nonequilibrium Seeded Air Flow in the NASA-Ames MHD Channel
}

Sumeet Gupta, John C. Tannehill, lowa State University, Ames, IA 50011

and

Unmeel B. Mehta

NASA Ames Research Center, Moffett Field, CA 94035

\section{3rd AIAA Aerospace Sciences Meeting and Exhibit 10-13 January 2005 / Reno, NV}




\title{
Simulation of 3-D Nonequilibrium Seeded Air Flow in the NASA-Ames MHD Channel
}

\author{
Sumeet Gupta* , John C. Tannehill ${ }^{\dagger}$ \\ Iowa State University, Ames, IA 50011 \\ and \\ Unmeel B. Mehta ${ }^{\ddagger}$ \\ NASA Ames Research Center, Moffett Field, CA 94035
}

\begin{abstract}
The 3-D nonequilibrium seeded air flow in the NASAAmes experimental MHD channel has been numerically simulated. The channel contains a nozzle section, a center section, and an accelerator section where magnetic and electric fields can be imposed on the flow. In recent tests, velocity increases of up to $40 \%$ have been achieved in the accelerator section. The flow in the channel is numerically computed using a 3-D parabolized Navier-Stokes (PNS) algorithm that has been developed to efficiently compute MHD flows in the low magnetic Reynolds number regime. The MHD effects are modeled by introducing source terms into the PNS equations which can then be solved in a very efficient manner. The algorithm has been extended in the present study to account for nonequilibrium seeded air flows. The electrical conductivity of the flow is determined using the program of Park. The new algorithm has been used to compute two test cases that match the experimental conditions. In both cases, magnetic and electric fields are applied to the seeded flow. The computed results are in good agreement with the experimental data.
\end{abstract}

\section{Introduction}

Magnetohyrodynamics (MHD) can be utilized to improve performance and extend the operational range of many systems. Potential applications include hypersonic cruise, advanced Earth-to-orbit propulsion, chemical and nuclear space propulsion, regenerative aerobraking, onboard flow control systems, test fa-

\footnotetext{
- Graduate Research Assistant, Student Member AIAA

†Manager, Computational Fluid Dynamics Center, and Professor, Dept. of Aerospace Engineering. Fellow AIAA

†Division Scientist, Associate Fellow, AlAA

Copyright (C)2005 by the American Institute of Aeronautics and Astronautics, Inc., all rights reserved.
}

cilities, launch assist, and power generation. One of the critical technologies associated with these applications is MHD acceleration. In order to study MHD acceleration, an experimental MHD channel has been built at NASA Ames Research Center by D. W. Bogdanoff, C. Park, and U. B. Mehta [1,2]. The channel is about a half meter long and contains a nozzle section, a center section, and an accelerator section. The channel has a uniform width of $2.03 \mathrm{~cm}$. Magnetic and electric fields can be imposed upon the flow in the accelerator section. A cross section of the MHD channel is shown in Fig. 1.

In the present study, the flow in the experimental MHD channel is numerically simulated. Flowfields involving MHD effects have typically been computed [3-15] by solving the complete NavierStokes (N-S) equations for fluid flow in conjunction with Maxwell's equations of electromagnetodynamics. When chemistry and turbulence effects are also included, the computational effort required to solve the resulting coupled system of partial differential equations is extremely formidable. One possible remedy to this problem is to use the parabolized NavierStokes (PNS) equations in place of the N-S equations. The PNS equations can be used to compute thre-dimensional, supersonic viscous flowfields in a very efficient manner [16]. This efficiency is achieved because the equations can be solved using a spacemarching technique as opposed to the time-marching technique that is normally employed for the complete $\mathrm{N}-\mathrm{S}$ equations.

Recently, the present authors have developed PNS codes to solve 2-D and 3-D supersonic MHD flowfields in both the high and low magnetic Reynolds number regimes [17-19]. The magnetic Reynolds number is defined as $R e_{m}=\sigma_{e} \mu_{e} V_{\infty} L$ where $\sigma_{e}$ is the electrical conductivity, $\mu_{e}$ is the magnetic permeability, $V_{\infty}$ is the freestream velocity, and $L$ is the reference length. The new MHD PNS codes are based on NASA's up- 
wind PNS (UPS) code which was originally developed by Lawrence et al. [20]. The UPS code solves the PNS equations using Roe's scheme in a fully conservative, finite-volume approach in general nonorthogonal coordinates.

For many aerospace applications, including the present experimental MHD channel, the electrical conductivity of the fluid is low and hence the magnetic Reynolds number is small. In these cases, it makes sense to use the low magnetic Reynolds number assumption and reduce the complexity of the governing equations. The MHD effects are modeled with the introduction of source terms into the PNS equations.

Previousiy [19], the present authors used the low magnetic Reynolds PNS code to compute both 2D and 3-D flows in the NASA-Ames MHD channel. These perfect gas $(\tilde{\gamma}=1.25)$ calculations assumed that the magnetic and electric fields, as well as the electrical conductivity, were constant in the accelerator section. In the present study, the 3-D simulations have been extended to include both equilibrium air flows as well as nonequilibrium seeded air flows. For the latter case, the electrical conductivity is variable and is computed using the program of Park [21].

\section{Governing Equations}

\section{Magnetogasdynamic Equations}

The governing equations for a viscous MHD flow with a small magnetic Reynolds number are given by [14]:

Continuity equation

$$
\frac{\partial \rho}{\partial t}+\nabla \cdot(\rho \mathbf{V})=0
$$

Momentum equation

$$
\frac{\partial(\rho \mathbf{V})}{\partial t}+\nabla \cdot[\rho \mathbf{V V}+p \overline{\overline{\mathbf{I}}}]=\nabla \cdot \overline{\bar{\tau}}+\mathbf{J} \times \mathbf{B}
$$

Energy equation

$$
\frac{\partial\left(\rho e_{t}\right)}{\partial t}+\nabla \cdot\left[\left(\rho e_{t}+p\right) \mathbf{V}\right]=\nabla \cdot(\mathbf{V} \cdot \overline{\bar{\tau}})-\nabla \cdot \mathbf{U}+\mathbf{E} \cdot \mathbf{J}(3)
$$

Ohm's law

$$
\mathbf{J}=\sigma_{e}(\mathbf{E}+\mathbf{V} \times \mathbf{B})
$$

where $\mathrm{V}$ is the velocity vector, $\mathrm{B}$ is the magnetic field vector, $\mathbf{E}$ is the electric field vector, and $\mathbf{J}$ is the conduction current density.
The governing magnetogasdynamic equations are nondimensionalized using the following reference variables:

$$
\begin{gathered}
x^{*}, y^{*}, z^{*}=\frac{x, y, z}{L}, \quad u^{*}, v^{*}, w^{*}=\frac{u, v, w}{U_{\infty}}, \quad t^{*}=\frac{U_{\infty} t}{L} \\
\rho^{*}=\frac{\rho}{\rho_{\infty}}, \quad T^{*}=\frac{T}{T_{\infty}}, \quad p^{*}=\frac{p}{\rho_{\infty} U_{\infty}^{2}} \\
e_{i}^{*}=\frac{e_{t}}{U_{\infty}^{2}}, \quad \bar{\tau}^{*}=\frac{\bar{\tau} L}{\mu_{\infty} U_{\infty}}, \quad \mu^{*}=\frac{\mu}{\mu_{\infty}} \quad(5) \\
B_{x}^{*}, B_{y}^{*}, B_{z}^{*}=\frac{B_{x}, B_{y}, B_{z}}{U_{\infty} \sqrt{\mu_{e} \rho_{\infty}}}, \quad E_{x}^{*}, E_{y}^{*}, E_{z}^{*}=\frac{E_{x}, E_{y}, E_{z}}{U_{\infty}^{2} \sqrt{\mu_{e} \rho_{\infty}}} \\
\mu_{e}^{*}=\frac{\mu_{e}}{\mu_{e}}=1, \quad \sigma_{e}^{*}=\frac{\sigma_{e}}{\sigma_{e \infty}}
\end{gathered}
$$

where the superscript $*$ refers to the nondimensional quantities. For convenience, the asterisks are dropped in the following equations.

The governing equations written in vector form in a 3-D Cartesian coordinate system become

$$
\frac{\partial \mathbf{U}}{\partial t}+\frac{\partial \mathbf{E}_{i}}{\partial x}+\frac{\partial \mathbf{F}_{i}}{\partial y}+\frac{\partial \mathbf{G}_{i}}{\partial z}=\frac{\partial \mathbf{E}_{v}}{\partial x}+\frac{\partial \mathbf{F}_{v}}{\partial y}+\frac{\partial \mathbf{G}_{v}}{\partial z}+\mathbf{S}_{\mathbf{M H D}}
$$

where $U$ is the vector of dependent variables, $\mathbf{E}_{\boldsymbol{i}}, \mathbf{F}_{\boldsymbol{i}}$ and $\mathbf{G}_{i}$ are the inviscid flux vectors, and $\mathbf{E}_{v}, \mathbf{F}_{v}$ and $\mathbf{G}_{v}$ are the viscous flux vectors. The source term $S_{M H D}$ contains all of the MHD effects. The flux vectors are given by

$$
\begin{gathered}
\mathrm{U}=\left[\begin{array}{cc}
\rho, \rho u, & \left.\rho v, \quad \rho w, \quad \rho e_{t}\right]^{T} \\
\mathbf{E}_{i}=\left[\begin{array}{c}
\rho u \\
\rho u^{2}+p \\
\rho u v \\
\rho u w \\
\left(\rho e_{t}+p\right) u
\end{array}\right], \mathrm{F}_{i}=\left[\begin{array}{c}
\rho v \\
\rho u v \\
\rho v^{2}+p \\
\rho v w \\
\left(\rho e_{t}+p\right) v
\end{array}\right] \\
\mathbf{G}_{i}=\left[\begin{array}{c}
\rho w \\
\rho u w \\
\rho v w \\
\rho w^{2}+p \\
\left(\rho e_{t}+p\right) w
\end{array}\right] \\
\mathbf{E}_{v}=\left[\begin{array}{c}
7 \\
0 \\
\tau_{x x} \\
\tau_{x y} \\
\tau_{x z} \\
\mathbf{F}_{v}
\end{array}\right] \\
0 \\
\tau_{y x} \\
\tau_{y y} \\
\tau_{y z} \\
u \tau_{x x}+v \tau_{x z}-q_{x}
\end{array}\right]
\end{gathered}
$$




$$
\begin{gathered}
\mathrm{G}_{v}=\left[\begin{array}{c}
0 \\
\tau_{z x} \\
\tau_{z y} \\
\tau_{z z} \\
u \tau_{z x}+v \tau_{z y}+w \tau_{z z}-q_{z}
\end{array}\right] \\
\mathrm{S}_{\mathrm{MHD}}=\operatorname{Re}_{m}\left[\begin{array}{c}
0 \\
\hline B_{z}\left(E_{y}+w B_{x}-u B_{z}\right) \\
-B_{y}\left(E_{z}+u B_{y}-v B_{x}\right) \\
\hline B_{x}\left(E_{z}+u B_{y}-v B_{x}\right) \\
-B_{z}\left(E_{x}+v B_{z}-w B_{y}\right) \\
\hline B_{y}\left(E_{x}+v B_{z}-w B_{y}\right) \\
-B_{x}\left(E_{y}+w B_{x}-u B_{z}\right) \\
\hline E_{x}\left(E_{x}+v B_{z}-w B_{y}\right) \\
+E_{y}\left(E_{y}+w B_{x}-u B_{z}\right) \\
+E_{z}\left(E_{z}+u B_{y}-v B_{x}\right)
\end{array}\right]
\end{gathered}
$$

where

$$
\rho e_{t}=\rho\left[e+\frac{1}{2}\left(u^{2}+v^{2}+w^{2}\right)\right]
$$

and the nondimensional shear stresses and heat fluxes are defined in the usual manner [16].

The governing equations are transformed into computational space and written in a generalized coordinate system $(\xi, \eta, \zeta)$ as

$$
\frac{1}{J} \mathbf{U}_{t}+\tilde{\mathbf{E}}_{\xi}+\tilde{\mathbf{F}}_{\eta}+\tilde{\mathbf{G}}_{\zeta}=\frac{\mathbf{S}_{M H D}}{J}
$$

where

$$
\begin{aligned}
\tilde{\mathbf{E}}= & \left(\frac{\xi_{x}}{J}\right)\left(\mathbf{E}_{i}-\mathbf{E}_{v}\right)+\left(\frac{\xi_{y}}{J}\right)\left(\mathbf{F}_{i}-\mathbf{F}_{v}\right) \\
& +\left(\frac{\xi_{z}}{J}\right)\left(\mathbf{G}_{i}-\mathbf{G}_{v}\right) \\
\tilde{\mathbf{F}}= & \left(\frac{\eta_{x}}{J}\right)\left(\mathbf{E}_{i}-\mathbf{E}_{v}\right)+\left(\frac{\eta_{y}}{J}\right)\left(\mathbf{F}_{i}-\mathbf{F}_{v}\right) \\
& +\left(\frac{\eta_{z}}{J}\right)\left(\mathbf{G}_{i}-\mathbf{G}_{v}\right) \\
\tilde{\mathbf{G}}= & \left(\frac{\zeta_{x}}{J}\right)\left(\mathbf{E}_{i}-\mathbf{E}_{v}\right)+\left(\frac{\zeta_{y}}{J}\right)\left(\mathbf{F}_{i}-\mathbf{F}_{v}\right) \\
& +\left(\frac{\zeta_{z}}{J}\right)\left(\mathbf{G}_{i}-\mathbf{G}_{v}\right)
\end{aligned}
$$

and $J$ is the Jacobian of the transformation.

The governing equations are parabolized by dropping the time derivative term and the streamwise direction $(\xi)$ viscous flow terms in the flux vectors. Equation (14) can then be rewritten as

$$
\tilde{\mathbf{E}}_{\xi}+\tilde{\mathbf{F}}_{\eta}+\tilde{\mathbf{G}}_{\zeta}=\frac{\mathbf{S}_{\mathrm{MHD}}}{J}
$$

where

$$
\begin{aligned}
\tilde{\mathbf{E}}= & \left(\frac{\xi_{x}}{J}\right) \mathbf{E}_{i}+\left(\frac{\xi_{y}}{J}\right) \mathbf{F}_{i}+\left(\frac{\xi_{z}}{J}\right) \mathbf{G}_{i} \\
\tilde{\mathbf{F}}= & \left(\frac{\eta_{x}}{J}\right)\left(\mathbf{E}_{i}-\mathbf{E}_{v}^{\prime}\right)+\left(\frac{\eta_{y}}{J}\right)\left(\mathbf{F}_{i}-\mathbf{F}_{v}^{\prime}\right) \\
& +\left(\frac{\eta_{z}}{J}\right)\left(\mathbf{G}_{i}-\mathbf{G}_{v}^{\prime}\right) \\
\tilde{\mathbf{G}}= & \left(\frac{\zeta_{x}}{J}\right)\left(\mathbf{E}_{i}-\mathbf{E}_{v}^{\prime}\right)+\left(\frac{\zeta_{y}}{J}\right)\left(\mathbf{F}_{i}-\mathbf{F}_{v}^{\prime}\right) \\
& +\left(\frac{\zeta_{z}}{J}\right)\left(\mathbf{G}_{i}-\mathbf{G}_{v}^{\prime}\right)
\end{aligned}
$$

The primes in the preceding equations indicate that the streamwise viscous flow terms have been dropped.

For turbulent flows, the two-layer Baldwin-Lomax turbulence model [22] has been modified to account for MHD effects. Only the expression for turbulent viscosity in the inner layer is changed. This modification for MHD flows is due to Lykoudis [23].

In order to "close" the preceding system of PNS equations, relations between the thermodynamic variables are required along with expressions for the transport properties $\mu$ and $k$. For a perfect gas, the pressure is computed from the relation

$$
p=(\tilde{\gamma}-1) \rho e
$$

where $\tilde{\gamma}=\gamma_{\infty}$, and the transport properties are computed using Sutherland's formulas [16]. For equilibrium air computations, $\tilde{\gamma}$ and all other thermodynamic and transport properties are obtained from the simplified curve fits of Srinivasan et al. [24,25]. For nonequilibrium computations, the thermodynamic and transport properties are determined using the procedures described in the next section.

\section{Nonequilibrium Flow Equations}

For nonequilibrium flows, the species continuity equations must be solved in addition to the magnetogasdynamic equations given previously. The magnetogasdynamic equations remain the same except for the additional term in the energy equation, which is due to the diffusion of the species. The nondimensional species continuity equations, expressed in 2-D transformed coordinates for a steady flow, are given by

$$
\begin{array}{r}
\rho u\left[\frac{\partial c_{s}}{\partial \xi}+\left(\eta_{x}+\frac{v}{u} \eta_{y}\right) \frac{\partial c_{s}}{\partial \eta}\right]=\dot{\omega}_{s}+ \\
\frac{\eta_{y}}{R e_{\infty}} \frac{\partial}{\partial \eta}\left(\beta_{3} \rho D \eta_{y} \frac{\partial c_{s}}{\partial \eta}\right)+\frac{\eta_{x}}{R e_{\infty}} \frac{\partial}{\partial \eta}\left(\beta_{3} \rho D \eta_{x} \frac{\partial c_{s}}{\partial \eta}\right) \\
(s=1,2, \ldots, n)
\end{array}
$$

where $c_{s}$ is the mass fraction of species $\mathrm{s}, \dot{\omega}_{s}$ is the nondimensional production term, $D$ is the nondimensional binary diffusion coefficient, and $\beta_{3}=\frac{\rho_{\infty} D_{\infty}}{\mu_{\infty}}$. 
The chemical model used in the present calculations is similar to the clean-air model of Blottner et al. [26] and Prabhu et al. [27]. It consists of molecular oxygen $\left(\mathrm{O}_{2}\right)$, atomic oxygen $(O)$, molecular nitrogen $\left(N_{2}\right)$, atomic nitrogen $(N)$, nitric oxide $(N O)$, nitric oxide ion $\left(\mathrm{NO}^{+}\right)$and electrons $\left(e^{-}\right)$. The following reactions are considered between the constituent species.

$$
\begin{array}{ll}
\text { (1) } & O_{2}+M_{1} \rightleftharpoons 2 O+M_{1} \\
\text { (2) } & N_{2}+M_{2} \rightleftharpoons 2 N+M_{2} \\
\text { (3) } & N_{2}+N \rightleftharpoons 2 N+N \\
\text { (4) } & N O+M_{3} \rightleftharpoons N+O+M_{3} \\
\text { (5) } & N O+O \rightleftharpoons O_{2}+N \\
\text { (6) } & N_{2}+O \rightleftharpoons N O+N \\
\text { (7) } & N O \rightleftharpoons N O^{+}+e^{-}
\end{array}
$$

where $M_{1}, M_{2}, M_{3}$, are catalytic third bodies. The clean-air chemical model has 7 species $(n=7)$ and seven reactions $(m=7)$. In order to simulate the seeded air flow in the MHD channel, the potassium seeding reaction has been added to the above chemistry model. This reaction is the ionization of atomic potassium $(K)$ and is given by the following equation:

$$
\text { (8) } K+e^{-} \rightleftharpoons K^{+}+e^{-}+e^{-}
$$

Using the law of mass action, the nondimensional mass production rate of species $s$ is

$$
\begin{array}{r}
\dot{\omega}_{s}=M_{s} \sum_{k=1}^{m}\left(\nu_{k, s}^{\prime \prime}-\nu_{k, s}^{\prime}\right)\left[K_{f, k}(T) \prod_{r=1}^{n_{t}}\left[\rho \gamma_{r}\right]^{\nu_{k, r}^{\prime}}\right. \\
\left.-K_{b, k}(T) \prod_{r=1}^{n_{t}}\left[\rho \gamma_{r}\right]^{\nu_{k, r}^{\prime \prime}}\right]
\end{array}
$$

where $\gamma_{r}$ is the nondimensional mole-mass ratio of the reactants, $M_{s}$ is the molecular weight of species $\mathrm{s}, \nu_{k, s}^{\prime}$ and $\nu_{k, s}^{\prime \prime}$ are the stoichiometric coefficients and $n_{t}$ is the number of reactants. Further details on the reaction rates and the thermodynamic and transport properties can be found in Ref. [26]. The electrical conductivity is determined from the species mole fractions, along with the temperature, density, and pressure of the gas, using the program of Park [21].

\section{Numerical Method}

\section{Solution of PNS Equations}

The governing PNS equations with MHD source terms have been incorporated into NASA's upwind PNS (UPS) code [20]. These equations can be solved very efficiently using a single sweep of the flowfield for many applications. For cases where upstream (elliptic) effects are important, the flowfield can be computed using multiple streamwise sweeps with either the IPNS [28], TIPNS [29], or FBIPNS [30] algorithms. This iterative process is continued until the solution is converged.

For the iterative PNS (IPNS) method, the $\overline{\mathbf{E}}$ vector is split using the Vigneron parameter $(\omega)$ [31]. This parameter does not need to be changed for the present low magnetic Reynolds number formulation. In the previous high magnetic Reynolds number code [17] it was necessary to modify the Vigneron parameter to account for MHD effects. After splitting, the $\tilde{\mathbf{E}}$ vector can be written as:

$$
\tilde{\mathrm{E}}=\mathrm{E}^{*}+\mathbf{E}^{p}
$$

where

$$
\begin{aligned}
& \mathbf{E}^{*}=\frac{\xi_{x}}{J}\left[\begin{array}{c}
\rho u \\
\rho u^{2}+\omega p \\
\rho u v \\
\rho u w \\
\left(\rho e_{t}+p\right) u
\end{array}\right]+\frac{\xi_{y}}{J}\left[\begin{array}{c}
\rho v \\
\rho u v \\
\rho v^{2}+\omega p \\
\rho v w \\
\left(\rho e_{t}+p\right) v
\end{array}\right] \\
& +\frac{\xi_{z}}{J}\left[\begin{array}{c}
\rho w \\
\rho u w \\
\rho v w \\
\rho \omega^{2}+\omega p \\
\left(\rho e_{t}+p\right) w
\end{array}\right] \\
& \mathbf{E}^{p}=\frac{\xi_{x}}{J}\left[\begin{array}{c}
0 \\
(1-\omega) p \\
0 \\
0 \\
0
\end{array}\right]+\frac{\xi_{\nu}}{J}\left[\begin{array}{c}
0 \\
0 \\
(1-\omega) p \\
0 \\
0
\end{array}\right] \\
& +\frac{\xi_{z}}{J}\left[\begin{array}{c}
0 \\
0 \\
0 \\
(1-\omega) p \\
0
\end{array}\right]
\end{aligned}
$$

The streamwise derivative of $\tilde{\mathbf{E}}$ is then differenced using a backward difference for $\mathrm{E}^{*}$ and a forward difference for the "elliptic" portion ( $\left.E^{p}\right)$ :

$$
\left(\frac{\partial \tilde{\mathbf{E}}}{\partial \xi}\right)_{i+1}=\frac{1}{\Delta \xi}\left[\left(\mathbf{E}_{i+1}^{*}-\mathbf{E}_{i}^{*}\right)+\left(\mathbf{E}_{i+2}^{p}-\mathbf{E}_{i+1}^{p}\right)\right]
$$

where the subscript $(i+1)$ denotes the spatial index (in the $\xi$ direction) where the solution is currently being computed. The vectors $\mathbf{E}_{i+1}^{*}$ and $\mathbf{E}_{i+1}^{p}$ are then 
linearized in the following manner:

$$
\begin{aligned}
& \mathrm{E}_{i+1}^{*}=\mathrm{E}_{i}^{*}+\left(\frac{\partial \mathrm{E}^{*}}{\partial \mathrm{U}}\right)_{i}\left(\mathrm{U}_{i+1}-\mathrm{U}_{i}\right) \\
& \mathbf{E}_{i+1}^{p}=\mathrm{E}_{i}^{p}+\left(\frac{\partial \mathrm{E}^{p}}{\partial \mathrm{U}}\right)_{i}\left(\mathrm{U}_{i+1}-\mathrm{U}_{i}\right)
\end{aligned}
$$

The Jacobians can be represented by

$$
\begin{aligned}
& A^{*}=\frac{\partial \mathbf{E}^{*}}{\partial \mathbf{U}} \\
& A^{p}=\frac{\partial \mathbf{E}^{p}}{\partial \mathbf{U}}
\end{aligned}
$$

After substituting the above linearizations into Eq. (25), the expression for the streamwise gradient of $\tilde{E}$ becomes

$$
\begin{aligned}
\left(\frac{\partial \tilde{\mathrm{E}}}{\partial \xi}\right)_{i+1}= & \frac{1}{\Delta \xi}\left[\left(A_{i}^{*}-A_{i}^{p}\right)\left(\mathrm{U}_{i+1}-\mathrm{U}_{i}\right)\right. \\
& \left.+\left(\mathbf{E}_{i+2}^{p}-\mathbf{E}_{i}^{p}\right)\right]
\end{aligned}
$$

The final discretized form of the fluid flow equations with MHD source terms is obtained by substituting Eq. (28) into Eq. (16) along with the linearized expressions for the fluxes in the cross flow plane. The final expression becomes:

$$
\begin{aligned}
& {\left[\frac{1}{\Delta \xi}\left(A_{i}^{*}-A_{i}^{p}\right)+\frac{\partial}{\partial \eta}\left(\frac{\partial \tilde{\mathbf{F}}}{\partial \mathrm{U}}\right)_{i}\right.} \\
& \left.+\frac{\partial}{\partial \zeta}\left(\frac{\partial \tilde{\mathbf{G}}}{\partial \mathrm{U}}\right)_{i}\right]^{k+1}\left(\Delta \mathrm{U}_{i}\right)^{k+1}=\text { RHS }
\end{aligned}
$$

where

$$
\begin{aligned}
&\left(\Delta \mathrm{U}_{i}\right)^{k+1}=\left(\mathrm{U}_{i+1}-\mathrm{U}_{i}\right)^{k+1} \\
& \text { RHS }=-\frac{1}{\Delta \xi}\left[\left(\mathrm{E}_{i+2}^{p}\right)^{k}-\left(\mathbf{E}_{i}^{p}\right)^{k+1}\right]-\left(\frac{\partial \tilde{\mathbf{F}}}{\partial \eta}\right)_{i}^{k+1} \\
&-\left(\frac{\partial \tilde{\mathbf{G}}}{\partial \zeta}\right)_{i}^{k+1}+\left(\frac{\mathrm{S}_{\mathrm{MHD}}}{J}\right)_{i}^{k+1}
\end{aligned}
$$

and the superscript $k+1$ denotes the current iteration (i.e. sweep) level. In the preceding equation, the MHD source term, $\mathbf{S}_{\mathbf{M H D}}$, is treated explicitly since it is evaluated using the velocity at station i $\left(\mathbf{V}_{i}\right)$. For most cases, this will not degrade the accuracy of the solution since $\Delta \xi$ is small and the velocity changes slowly. If this is not the case, a predictor-corrector procedure can be implemented whereby a predicted velocity at station $i+1\left(\mathbf{V}_{\widetilde{i+1}}\right)$ is first obtained using Eq. (29). The solution at station $i+1$ is then recomputed by evaluating $\mathbf{S}_{\text {MHD }}$ with $\mathbf{V}_{\overparen{i+1}}$.

\section{Solution of Species Continuity Equations}

For chemical nonequilibrium, the species continuity equations, Eq. (19), must be solved in addition to the magnetogasdynamic equations. The equations have been integrated using the loosely-coupled approach of Tannehill et al. [32]. In this approach, the species continuity equations and magnetogasdynamic equations are solved separately. The coupling between the two sets of equations is then obtained in an approximate manner. The species continuity equations are modeled using a second-order-accurate, upwind-based TVD scheme for the convective terms and second-order-accurate central differences for the diffusion terms. The assumption of zero net charge of the gas is used to eliminate the electron mass conservation equation. In addition, the species continuity equation for the $n$th species is eliminated by using the requirement that the mass fractions must sum to unity. The term representing the rate of production of species, $\dot{w}_{s}$, is treated as a source term, and is lagged to the previous marching level.

The coupling between the fluids and the chemistry is performed in an approximate manner. First, a fluid step is taken from marching station $i$ to $i+1$ assuming frozen chemistry. Then the fluid density and velocity at $i+1$ are used in the solution of the species continuity equations to obtain species mass fractions at $i+1$. Finally, the species mass fractions, molecular weight of mixture, fluid density, and internal energy at i+1 are used to obtain the new temperature, pressure, specific enthalpy, and frozen specific heats at the $i+1$ marching station.

The temperature is obtained by performing a Newton-Raphson iteration of the following form:

$$
T^{k+1}=T^{k}-\frac{g\left(T^{k}\right)-h}{g^{\prime}\left(T^{k}\right)}
$$

where

$$
\begin{gathered}
g(T)=\sum_{s=1}^{n} c_{s} h_{s}(T) \\
g^{\prime}(T)=\sum_{s=1}^{n} c_{s} C_{p, s}(T)
\end{gathered}
$$

and $k$ is the index of iteration. The iterations are continued until

$$
\left|T^{k+1}-T^{k}\right| \leq \epsilon
$$

where $\epsilon$ is a small positive quantity. Once the temperature is determined, the pressure can be computed using Dalton's law of partial pressures. Further details of this procedure can be found in Refs. [32] and [33]. 


\section{Numerical Results}

The numerical calculation of the 3-D supersonic flow in the experimental MHD channel is now discussed. The flow in the nozzle section was computed using a combination of the OVERFLOW code [34] and the present PNS code (without MHD effects). For the OVERFLOW nozzle calculation, a highly stretched grid consisting of $150 \times 80 \times 80$ grid points was used. The normal grid spacing at the wall was $1.0 \times 10^{-5}$ m. For the PNS calculation of the flow in the remainder of the nozzle and the rest of the MHD channel, a highly stretched grid consisting of 90 points in both the $y$ and $z$ directions was used and the normal grid spacing at the wall was $2.0 \times 10^{-6} \mathrm{~m}$. As a consequence of flow symmetry, only one-fourth of the channel cross section was computed in the 3-D calculations.

The calculations were performed assuming turbulent flow throughout the MHD channel. The channel wall temperature was assumed to be isothermal since quasi-steady flow conditions were maintained in the experiment for only about 1.2 milliseconds. A schematic of the powered portion of the MHD channel along with the directions of the applied magnetic and electric fields is shown in Fig. 2. The values of the magnetic field $\left(B_{z}\right)$, and the electric field $\left(E_{y}\right)$ were kept constant in the powered portion of the channel.

Three different chemistry models were used in this study to simulate the flow in the MHD channel. These were: (1) perfect gas $(\bar{\gamma}=1.25)$, (2) equilibrium air, and (3) nonequilibrium seeded-air chemistry. For the perfect gas and equilibrium air calculations, the electrical conductivity $\left(\sigma_{e}\right)$ was assumed constant. For the nonequilibrium seeded-air calculations, the electrical conductivity varied throughout the flowfield and was determined using the program of Park [21]. The seeding (as in the experiment) consisted of $1 \%$ (by mass) of potassium. Two test cases corresponding to Runs 15 and 16 of the NASA Ames experiments $[2,35]$ were computed in this study and are now discussed.

Test Case 1: NASA Ames MHD Run 15

$$
\left(V_{\text {cap. }}=320 \mathrm{~V}\right)
$$

The dimensional flow parameters for this test case are:

$$
\begin{aligned}
p_{o} & =9.10 \times 10^{5} \mathrm{~N} / \mathrm{m}^{2} \\
T_{o} & =5560 \mathrm{~K} \\
T_{w} & =300 \mathrm{~K} \\
\sigma_{e} & =130 \mathrm{mho} / \mathrm{m} \text { (or variable) } \\
B_{z} & =0.0,0.92 \mathrm{~T} \\
E_{y} & =0,3955,5000 \mathrm{~V} / \mathrm{m}
\end{aligned}
$$

where the subscript o denotes total conditions at the nozzle entrance and $w$ denotes wall conditions.

This case was computed using several different electric field strengths in order to properly simulate the experiment. In the experiment, the voltage applied to the electrodes was approximately $134 \mathrm{~V}$ for this case, however, due to the sheath voltage drop, the actual voltage applied to the flow is smaller than the electrode voltage. The voltage drop was measured for the central inviscid core flow, and was approximately $67 \mathrm{~V}$ [2]. Since the boundary layer is computed in the numerical solution, the applied electric field must be approximately the voltage drop across the electrodes minus the sheath voltage drop. Unfortunately, it is not a trivial task to measure the sheath voltage drop. Therefore, several different electric field strengths were chosen in the numerical calculations so that the corresponding voltage drop across the electrodes would be between $67 \mathrm{~V}$ and $134 \mathrm{~V}$. The voltage drop of $67 \mathrm{~V}$ corresponds to $E_{y}=3955 \mathrm{~V} / \mathrm{m}$ and a voltage drop of $84.7 \mathrm{~V}$ corresponds to $E_{y}=5000 \mathrm{~V} / \mathrm{m}$.

The computed streamwise variation of static pressure for the nonequilibrium seeded-air calculations is shown in Fig. 3 for the different electric field strengths. The pressure variation with no electric field or magnetic field is denoted by $E_{y}=0$. The re sults for $E_{y}=5000 \mathrm{~V} / \mathrm{m}$ are in excellent agreement with the experiment. The numerical results show an increase in static pressure as the electric field strength is increased. The computed streamwise variation of static pressure for the different chemistry models is shown in Fig. 4 for $E_{y}=5000 \mathrm{~V} / \mathrm{m}$. The nonequilibrium seeded-air model gives the closest agreement with the experimental pressures.

For the nonequilibrium seeded-air computations, the electrical conductivity was not constant but varied throughout the flowfield. The average conductivity (averaged over the channel cross section) at the center of the powered portion of the channel (electrode pair 10) was found to be $130 \mathrm{mho} / \mathrm{m}$ for $E_{y}=5000 \mathrm{~V} / \mathrm{m}$. This value of conductivity is within the range determined in the experiments and is the same constant value that was used for the perfect gas and equilibrium air computations.

The computed streamwise variation of averaged velocity for the nonequilibrium seeded-air calculations is shown in Fig. 5. The velocities are averaged across the channel cross section and normalized using the entrance velocity to be consistent with the experiment. In the experiment, the velocities were obtained by measuring the voltage generated by the flow at the last electrode pair (19) which is unpowered. This procedure inherently involves an averaging of the velocity profile. The numerical results indicate an in- 
crease in the averaged velocity of about $27 \%$ with $E_{y}=5000 \mathrm{~V} / \mathrm{m}$ and this agrees exactly with the experimental value of $27 \%$.

The computed streamwise variation of averaged velocity for the different chemistry models is shown in Fig. 6 for $E_{y}=5000 \mathrm{~V} / \mathrm{m}$. Both the equilibrium air and the nonequilibrium seeded-air chemistry models give similar results. The centerline variation of static temperature for the nonequilibrium seeded-air model is given in Fig. 7 for the different electric field strengths

\section{Test Case 2: NASA Ames MHD Run 16} $\left(V_{\text {cap. }}=380 \mathrm{~V}\right)$

The dimensional flow parameters for this test case are:

$$
\begin{aligned}
p_{o} & =9.92 \times 10^{5} \mathrm{~N} / \mathrm{m}^{2} \\
T_{o} & =5560 \mathrm{~K} \\
T_{w} & =300 \mathrm{~K} \\
\sigma_{e} & =130 \mathrm{mho} / \mathrm{m} \text { (or variable) } \\
B_{z} & =0.0,0.92 \mathrm{~T} \\
E_{y} & =0,4309,5300,6000 \mathrm{~V} / \mathrm{m}
\end{aligned}
$$

This test case was also computed using several different electric field strengths in order to properly simulate the experiment. The electric field strength of $4309 \mathrm{~V} / \mathrm{m}$ corresponds to the voltage drop measured in the central inviscid core flow. The computed streamwise variation of static pressure for the nonequilibrium seeded-air calculations is shown in Fig. 8 for the different electric field strengths. The experimental pressure variation agrees with the numerical result with an electric field strength of $5300 \mathrm{~V} / \mathrm{m}$. The computed streamwise variation of static pressure for the different chemistry models is shown in Fig. 9 for $E_{y}=6000 \mathrm{~V} / \mathrm{m}$. The different chemistry models produce similar results.

The averaged electrical conductivity at the center of the powered portion of the channel (electrode pair 10) was found to be $142 \mathrm{mho} / \mathrm{m}$ for $E_{y}=5300 \mathrm{~V} / \mathrm{m}$. This value is at the upper end of the range determined in the experiments and is higher than the constant value of $130 \mathrm{mho} / \mathrm{m}$ used in the perfect gas and equilibrium air computations.

The computed streamwise variation of averaged velocity for the nonequilibrium seeded-air calculations is shown in Fig. 10. The numerical results indicate an increase in the averaged velocity of about $38 \%$ for $E_{y}=6000 \mathrm{~V} / \mathrm{m}$ and this agrees closely with the experimental value of $39 \%$. The computed streamwise variation of averaged velocity for the different chemistry models is shown in Fig. 11 for $E_{y}=6000 \mathrm{~V} / \mathrm{m}$. Once again, the equilibrium air and the nonequilibrium seeded-air models give similar results. The centerline variation of static temperature for the equilibrium model is shown in Fig. 12 for the different electric field strengths.

\section{Concluding Remarks}

In this study, a new 3-D parabolized Navier-Stokes algorithm with nonequilibrium seeded-air capability has been developed to efficiently compute MHD flows in the low magnetic Reynolds number regime. The new algorithm has been used to compute the flow in the NASA-Ames experimental MHD channel for Runs 15 and 16. The numerical results are in good agreement with the experimental results.

\section{Acknowledgments}

This work was supported by NASA Ames Research Center under Grant NCC2-5517 and by Iowa State University. The Technical Monitor for this grant is Dr. Unmeel B. Mehta. The authors wish to thank Dr. David W. Bogdanoff of NASA Ames for his help and comments regarding the MHD channel experiments and Prof. Robert W. MacCormack of Stanford University for his assistance with the electrical conductivity program of Dr. Chul Park.

\section{References}

[1] Bogdanoff, D. W., Park, C., and Mehta, U. B., "Experimental Demonstration of MagnetoHydrodynamic (MHD) Acceleration - Facility and Conductivity Measurements," NASA TM2001-210922, July 2001.

[2] Bogdanoff, D. W. and Mehta, U. B., "Experimental Demonstration of Magneto-HydroDynamic (MHD) Acceleration," AIAA Paper 2003-4285, June 2003.

[3] Gaitonde, D. V., "Development of a Solver for 3D Non-Ideal Magnetogasdynamics," AIAA Paper 99-3610, June 1999.

[4] Damevin, H. M., Dietiker, J.-F., and Hoffmann, K. A., "Hypersonic Flow Computations with Magnetic Field," AIAA Paper 2000-0451, Jan. 2000. 
[5] Hoffmann, K. A., Damevin, H. M., and Dietiker, J.-F., "Numerical Simulation of Hypersonic Magnetohydrodynamic Flows," AIAA Paper 2000-2259, June 2000.

[6] Gaitonde, D. V. and Poggie, J., "Simulation of MHD Flow Control Techniques," ALAA Paper 2000-2326, June 2000.

[7] MacCormack, R. W., "Numerical Computation in Magnetofluid Dynamics," Computational Fluid Dymamics for the 21st Century, Kyoto, Japan, July 2000.

[8] Deb, P. and Agarwal, R., "Numerical Study of MHD-Bypass Scramjet Inlets with Finite-Rate Chemistry," AlAA Paper 2001-0794, Jan. 2001.

[9] MacCormack, R. W., "A Computational Method for Magneto-Fluid Dynamics," AIAA Paper 2001-2735, June 2001.

[10] Gaitonde, D. V. and Poggie, J., "An Implicit Technique for 3-D Turbulent MGD with the Generalized Ohm's Law," AIAA Paper 20012736, June 2001.

[11] Dietiker, J.-F. and Hoffmann, K. A., Numerical Simulation of Turbulent Magnetohydrodynamic Flows," ALAA Paper 2001-2737, June 2001.

[12] MacCormack, R. W., "Three Dimensional Magneto-Fluid Dynamics Algorithm Development," AIAA Paper 2002-0197, Jan. 2002.

[13] Munipalli, R., Anderson, D. A., and Kim, H., "Two-Temperature Computations of Ionizing Air with MHD Effects," AIAA Paper 2000-0450, Jan. 2000.

[14] Dietiker, J.-F. and Hoffmann, K. A., "Boundary Layer Control in Magnetohydrodynamic Flows," AIAA Paper 2002-0130, Jan. 2002.

[15] Cheng, F., Zhong, X., Gogineni, S., and Kimmel, R. L., "Effect of Applied Magnetic Field on the Instability of Mach 4.5 Boundary Layer over a Flat Plate," AIAA Paper 2002-0351, Jan. 2002.

[16] Tannehill, J. C., Anderson, D. A., and Pletcher, R. H., Computational Fluid Mechanics and Heat Transfer, Taylor and Francis, Washington, D.C., 1997.

[17] Kato, H., Tannehill, J. C., Ramesh, M. D., and Mehta, U. B., "Computation of Magnetohydrodynamic Flows Using an Iterative PNS Algorithm," AIAA Paper 2002-0202, Jan. 2002.
[18] Kato, H., Tannehill, J. C., and Mehta, U. B., "Numerical Simulation of Turbulent MHD Flows Using an Iterative PNS Algorithm," AIAA Paper 2003-0326, Jan. 2003.

[19] Kato, H., Tannehill, J. C., Gupta, S., and Mehta, U. B., "Numerical Simulation of 3-D Supersonic, Viscous Flow in an Experimental MHD Channel," AIAA Paper 2004-0317, Jan. 2004.

[20] Lawrence, S. L., Tannehill, J. C., and Chaussee, .D. S., "Upwind Algorithm for the Parabolized Navier-Stokes Equations," AIAA Journal, Vol. 27, No. 9, Sept. 1989, pp. 1975-1983.

[21] Park, C., Mehta, U. B., and Bogdanoff, D. W., "Magnetohydrodynamics Energy Bypass Scramjet Performance with Real Gas Effects," Journal of Propulsion and Power, Vol. 17, No. 5, 2001, pp. 1049-1057.

[22] Baldwin, B. S. and Lomax, H., "Thin Layer Approximation and Algebraic Model for Separated Turbulent Flows," AIAA-Paper 78-257, Jan. 1978.

[23] Lykoudis, P. S., "Magneto Fluid Mechanics Channel Flow, II Theory," The Physics of Fluids, Vol. 10, No. 5, May 1967, pp. 1002-1007.

[24] Srinivasan, S., Tannehill, J. C., and Weilmuenster, K. J., "Simplified Curve Fits for the Thermodynamic Properties of Equilibrium Air," NASA RP 1181, Aug. 1987.

[25] Srinivasan, S., Tannehill, J. C., and Weilmuenster, K. J., "Simplified Curve Fits for the Transport Properties of Equilibrium Air," NASA CR 178411 , Dec. 1987.

[26] Blottner, F. G., Johnson, M., and Ellis, M., "Chemically Reacting Viscous Flow Program for Multi-Component Gas Mixtures," Sandia Labs., Albuquerque, NM Rept. SC-RR-70-754, Dec. 1971.

[27] Prabhu, D. K., Tannehill, J. C., and Marvin, J. G., "A new PNS Code for Three-Dimensional Chemically Reacting Flows," Journal of Thermophysics and Heat Transfer, Vol. 4, No. 3, 1990, pp. 257-258.

[28] Miller, J. H., Tannehill, J. C., and Lawrence, S. L., "PNS Algorithm for Solving Supersonic Flows with Upstream Influences," ALAA Paper 98-0226, Jan. 1998. 
[29] Tannehill, J. C., Miller, J. H., and Lawrence, S. L., "Development of an Iterative PNS Code for Separated Flows," AIAA Paper 99-3361, June 1999.

[30] Kato, H. and Tannehill, J. C., "Development of a Forward-Backward Sweeping Parabolized Navier-Stokes Algorithm," AIAA Paper 20020735, Jan. 2002.

[31] Vigneron, Y. C., Rakich, J. V., and Tannehill, J. C., "Calculation of Supersonic Flow over Delta Wings with Sharp Subsonic Leading Edges," ALAA Paper 78-1137, July 1978.

[32] Tannehill, J. C., Ievalts, J. O., Buelow, P. E., Prabhu, D. K., and Lawrence, S. L., "Upwind Parabolized Navier-Stokes Code for Chemically Reacting Flows," Journal of Thermophysics and Heat Transfer, Vol. 4, No. 2, 1990, pp. 149-156.

[33] Buelow, P. E., Tannehill, J. C., Ievalts, J. O., and Lawrence, S. L., "A Three-Dimensional Upwind Parabolized Navier-Stokes Code for Chemically Reacting Flows," Journal of Thermophysics and Heat Transfer, Vol. 5, No. 3, JulySept. 1991, pp. 274-283.

[34] Buning, P. G., Jespersen, D. C., and Pulliam, T. H., OVERFLOW Manual, Version 1.7v, NASA Ames Research Center, Moffett Field, California, June 1997.

[35] Bogdanoff, D. W., "Private Communication," Dec. 2003. 


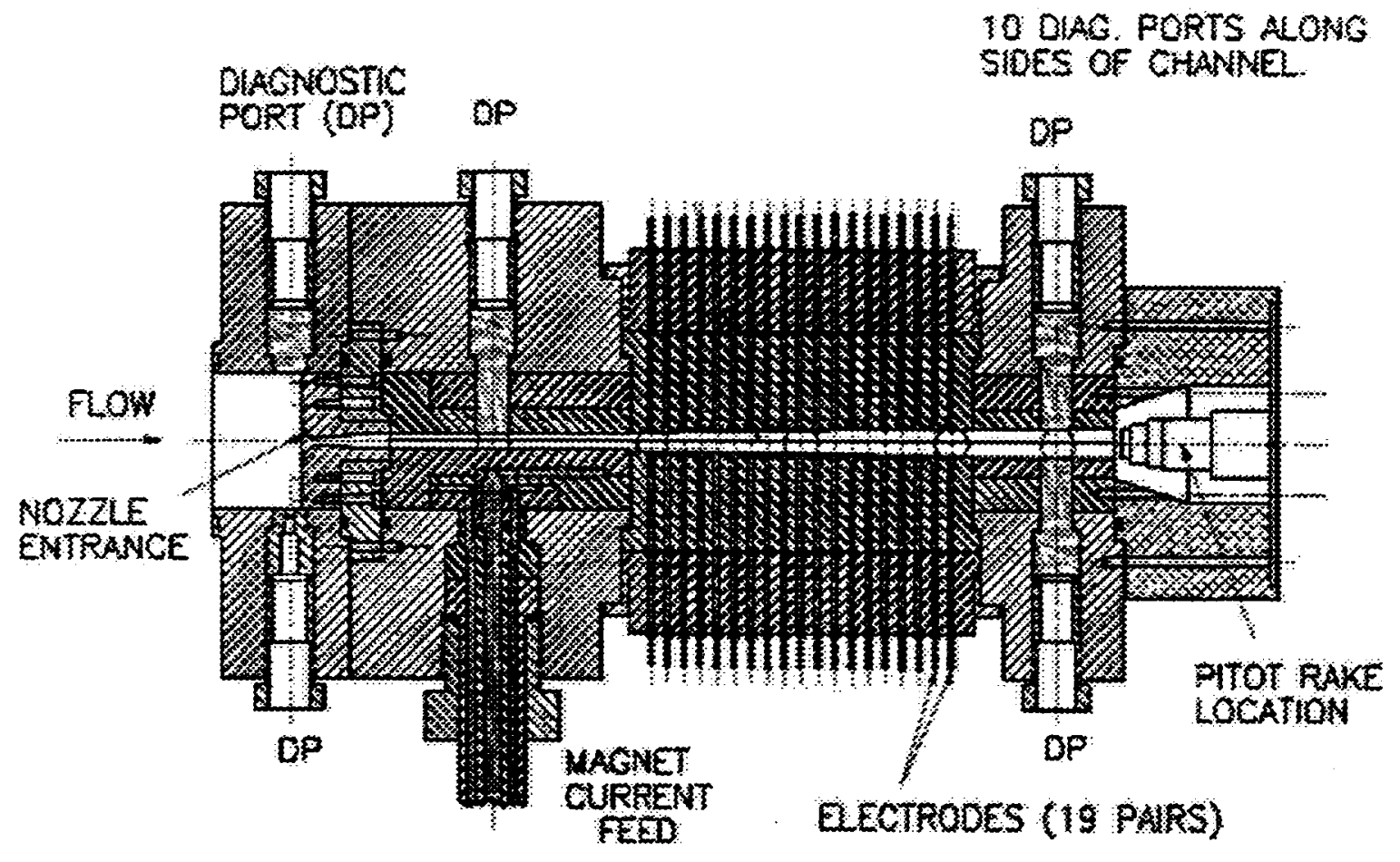

Figure 1: Cross section of NASA Ames MHD Channel

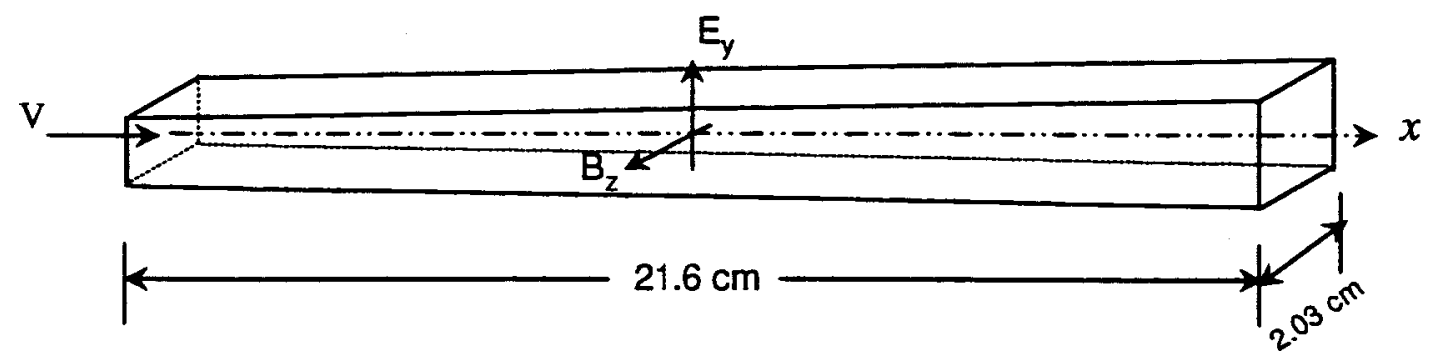

Figure 2: Schematic of powered portion of MHD channel 


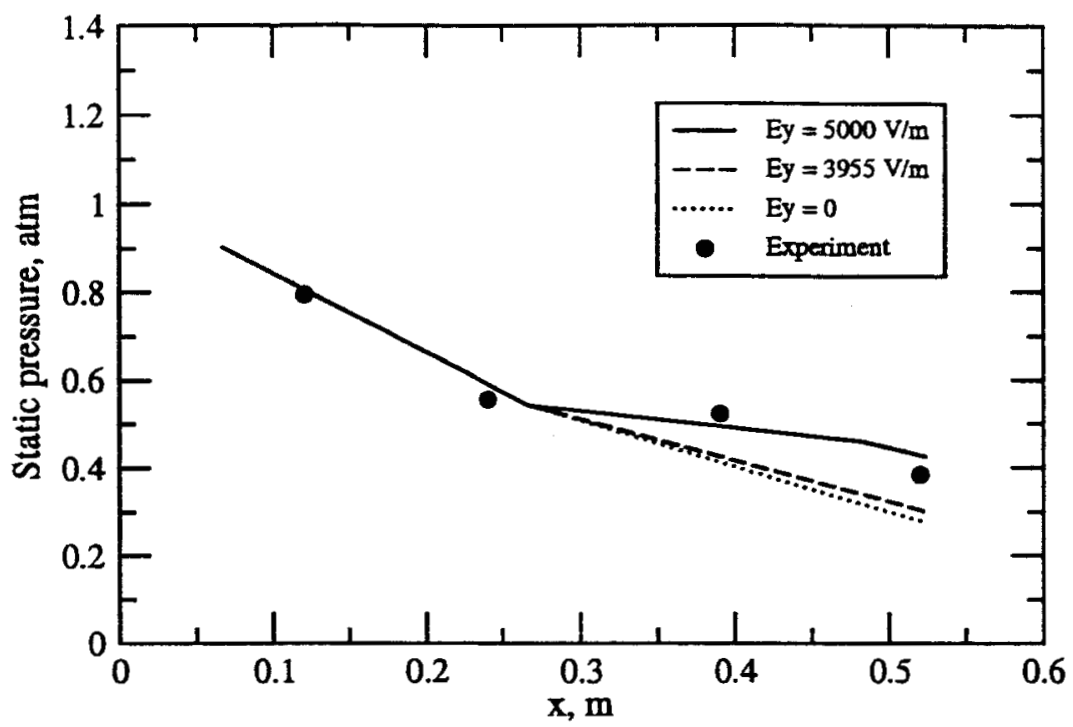

Figure 3: Streamwise variation of static pressure for Run 15 (seeded-air model)

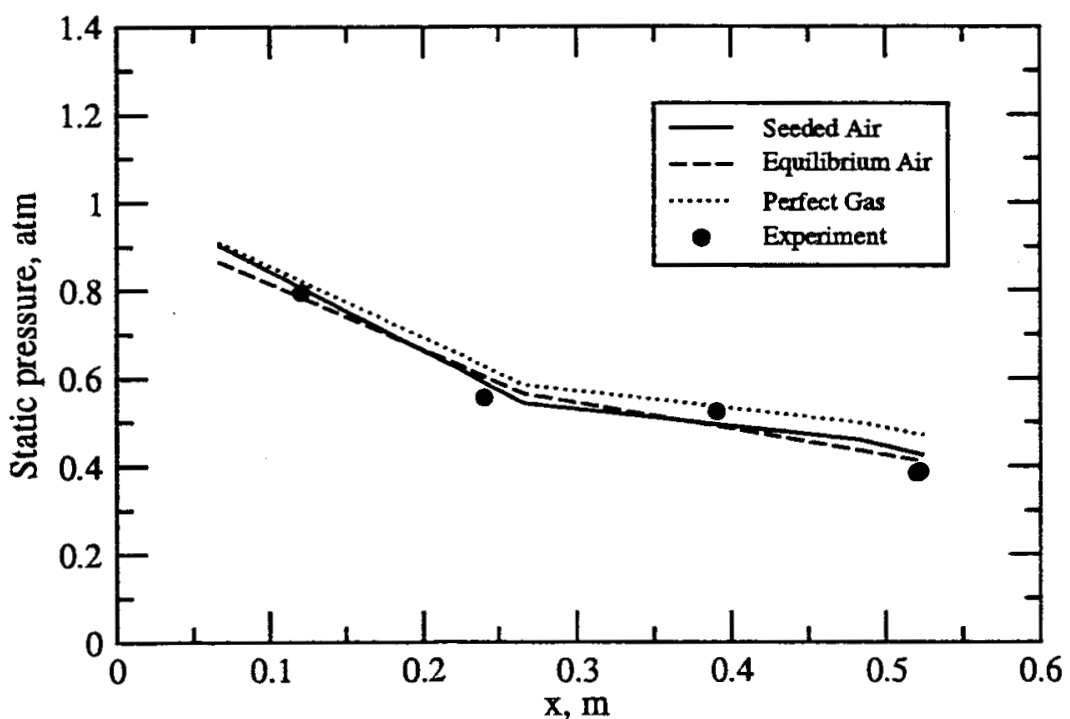

Figure 4: Streamwise variation of static pressure for Run $15\left(E_{y}=5000 \mathrm{~V} / \mathrm{m}\right)$ 


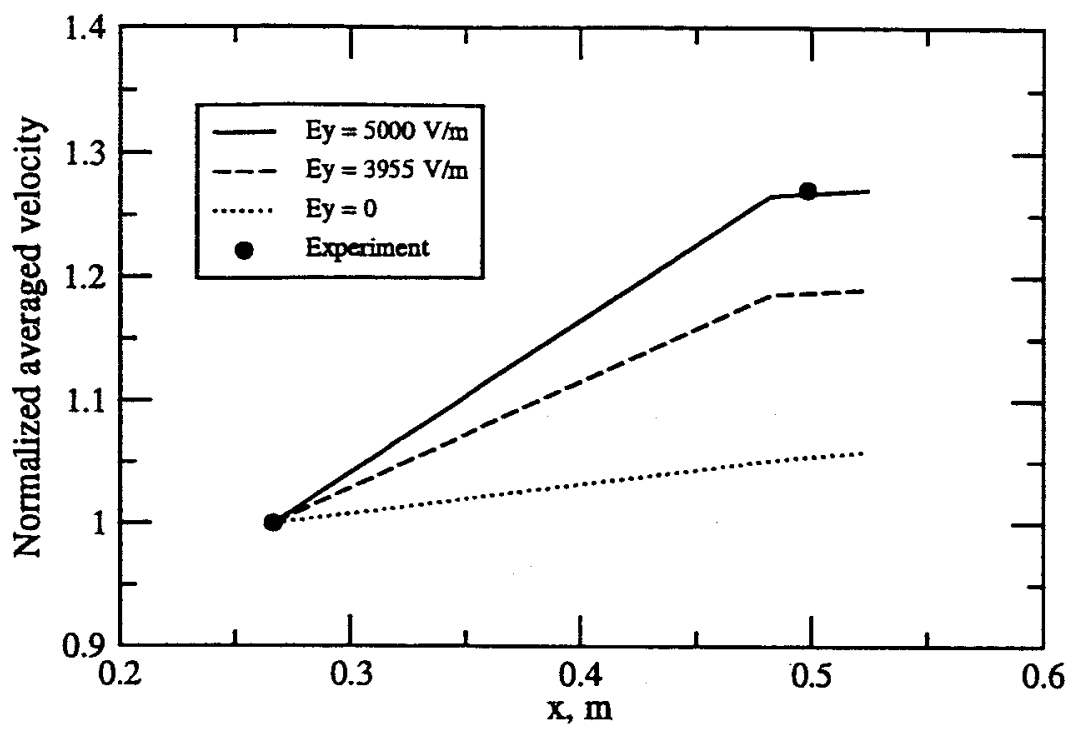

Figure 5: Streamwise variation of averaged velocity for Run 15 (seeded-air model)

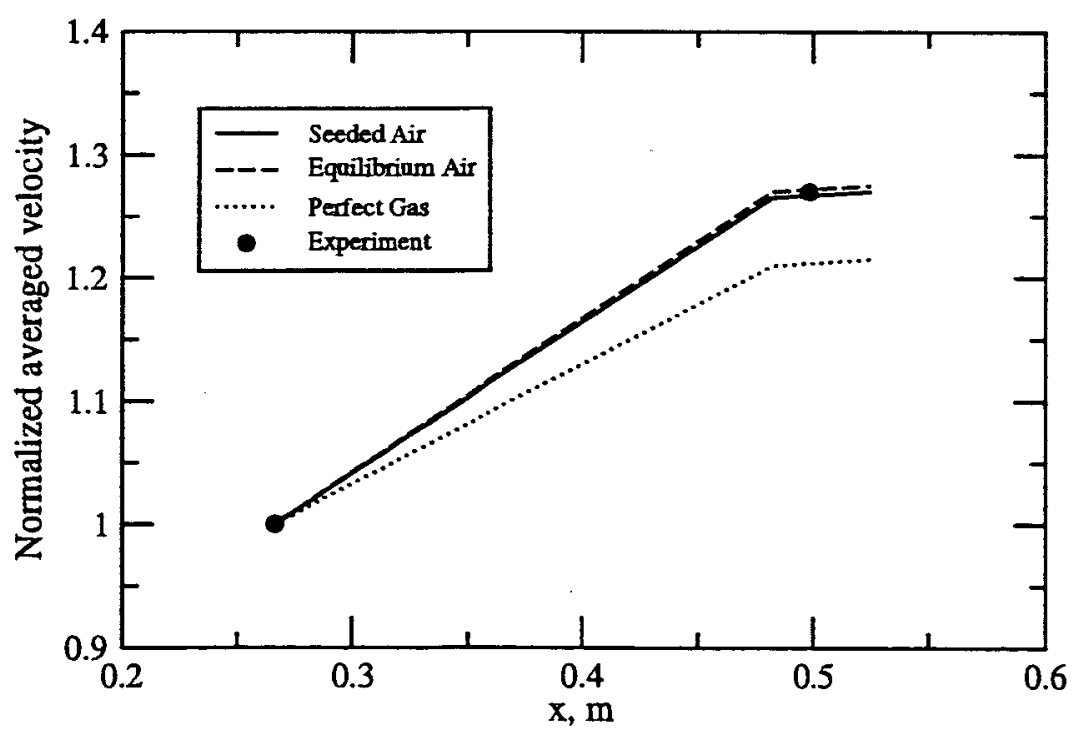

Figure 6: Streamwise variation of averaged velocity for Run $15\left(E_{y}=5000 \mathrm{~V} / \mathrm{m}\right)$ 


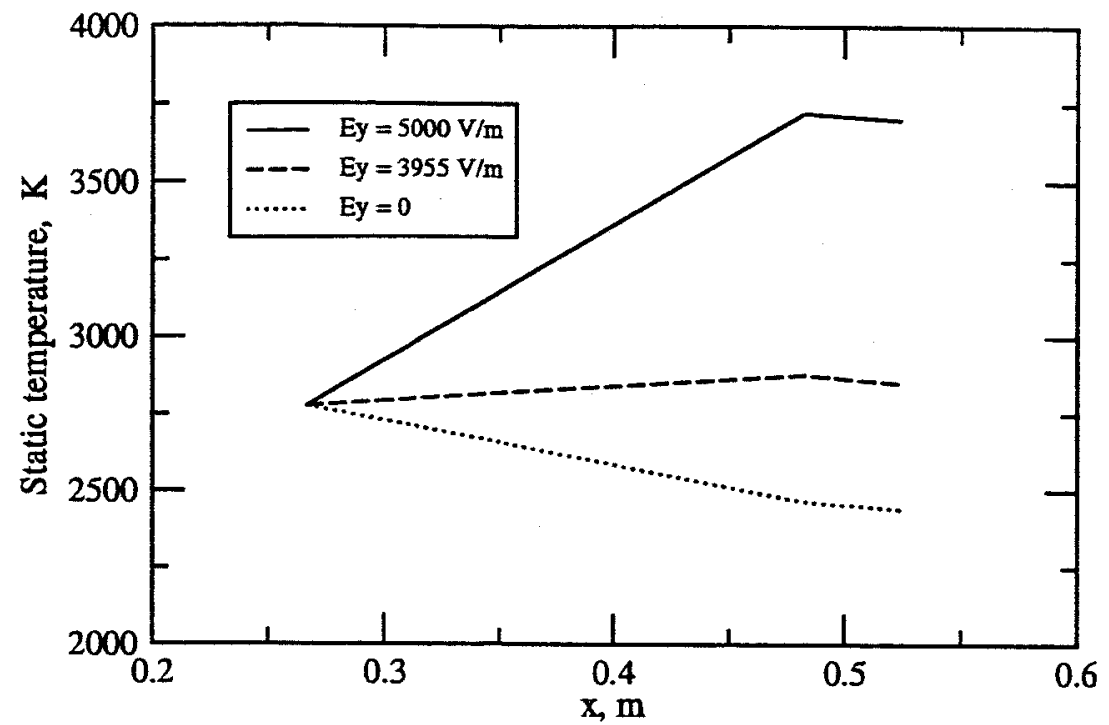

Figure 7: Streamwise variation of static temperature for Run 15 (seeded-air model)

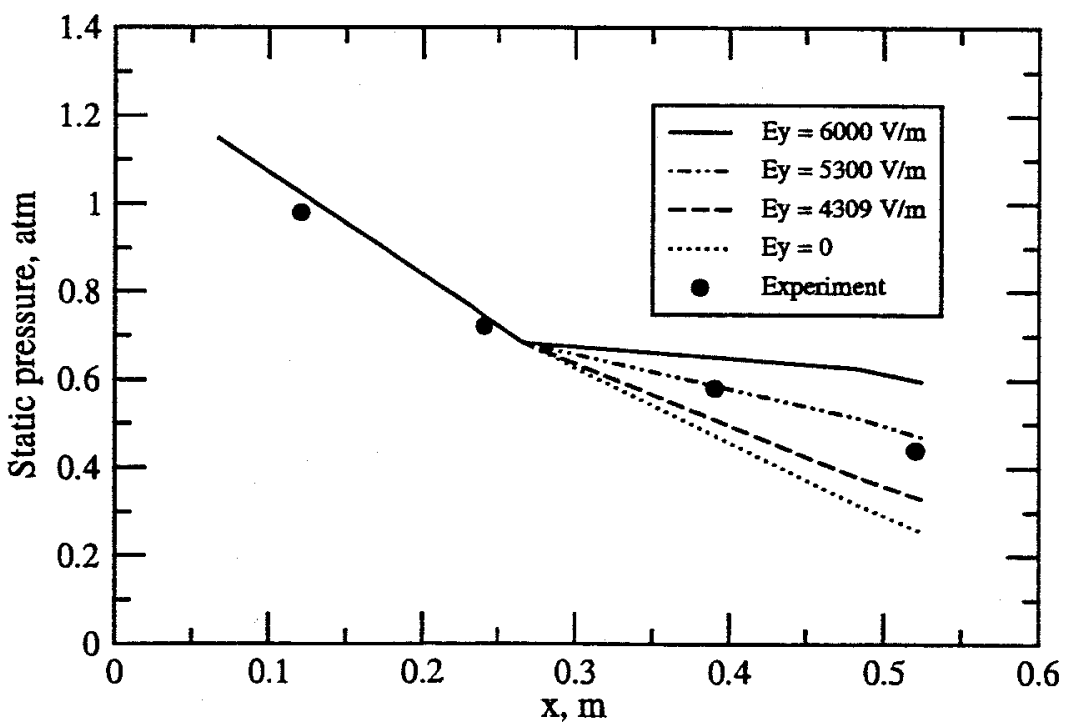

Figure 8: Streamwise variation of static pressure for Run 16 (seeded-air model) 


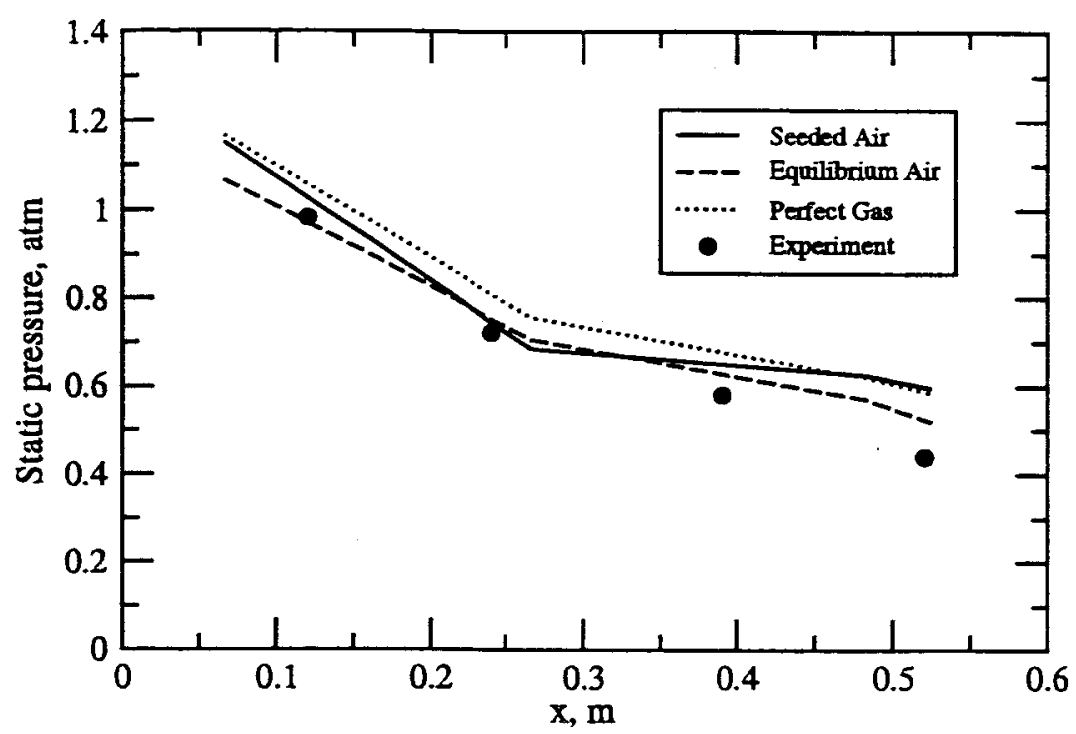

Figure 9: Streamwise variation of static pressure for Run $16\left(E_{y}=6000 \mathrm{~V} / \mathrm{m}\right)$

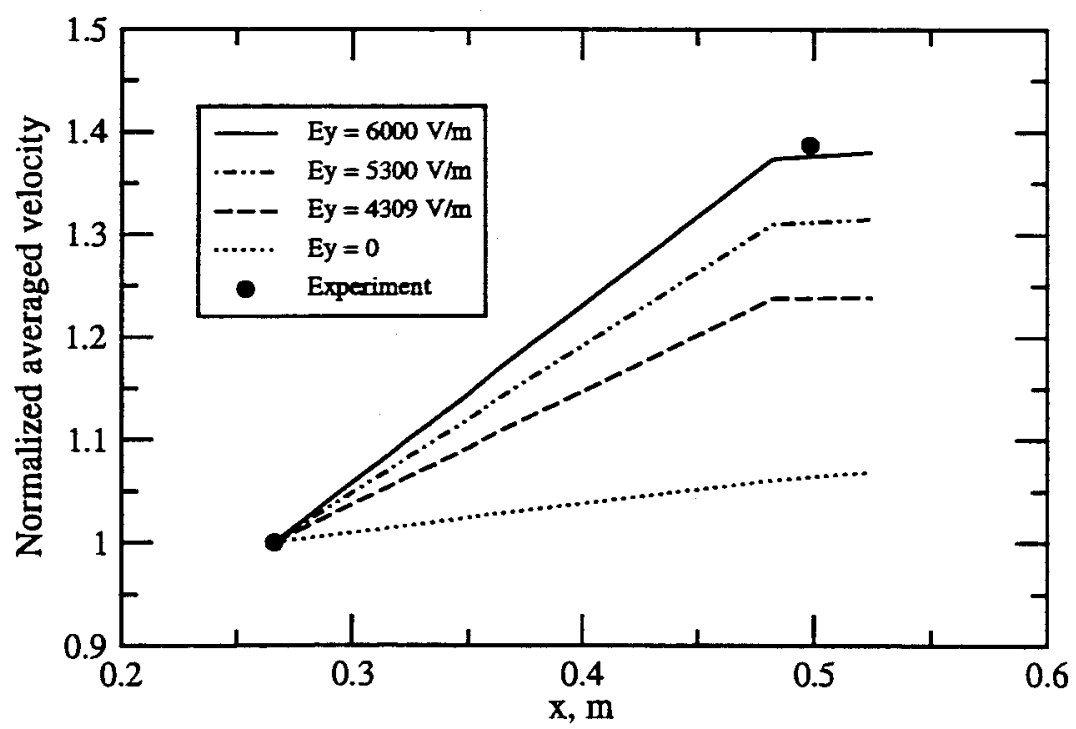

Figure 10: Streamwise variation of averaged velocity for Run 16 (seeded-air model) 


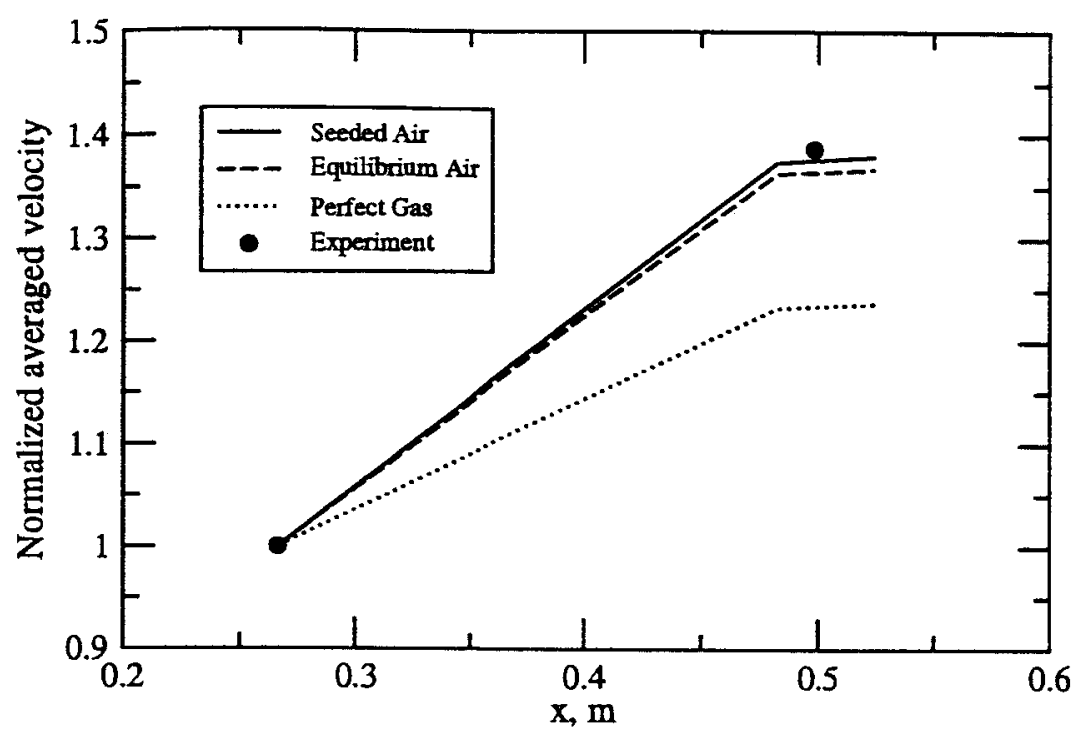

Figure 11: Streamwise variation of averaged velocity for Run $16\left(E_{y}=6000 \mathrm{~V} / \mathrm{m}\right)$

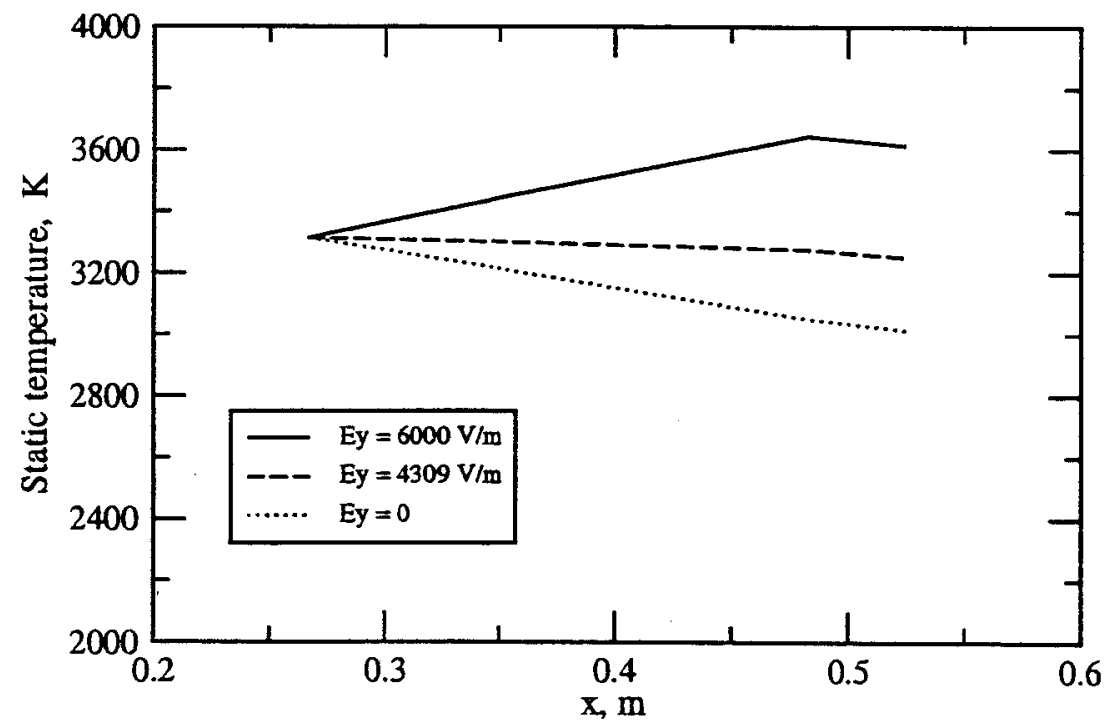

Figure 12: Streamwise variation of static temperature for Run 16 (equilibrium air model) 